\title{
A simple and versatile phase detector for heterodyne interferometers
}

\author{
A. Mlynek ${ }^{1}$, H. Faugel ${ }^{1}$, H. Eixenberger ${ }^{1}$, G. Pautasso ${ }^{1}$, G. Sellmair ${ }^{1}$, and the ASDEX \\ Upgrade Team ${ }^{\mathrm{a}}$ ) \\ ${ }^{1}$ Max Planck Institute for Plasma Physics, Garching 85748, Germany
}

The measurement of the relative phase of two sinusoidal electrical signals is a frequently encountered task in heterodyne interferometry, but also occurs in many other applications. Especially in interferometry, multi-radian detectors are often required, which track the temporal evolution of the phase difference and are able to register phase changes that exceed $2 \pi$. While a large variety of solutions to this problem is already known, we present an alternative approach, which pre-processes the signals with simple analog circuitry and digitizes two resulting voltages with an analog-todigital converter (ADC), whose sampling frequency can be far below the frequency of the sinusoidal signals. Phase reconstruction is finally carried out by software. The main advantage of this approach is its simplicity, using only few low-cost hardware components and a standard 2-channel ADC with low performance requirements. We present an application on the two-color interferometer of the ASDEX Upgrade tokamak, where the relative phase of $40 \mathrm{MHz}$ sinusoids is measured.

a) For authors' list, see U. Stroth et al., Nucl. Fusion 53, 104003 (Year: 2013). 


\section{INTRODUCTION}

In interferometry, the phase of an electromagnetic wave after passing through a refractive medium is measured, often relatively to an external reference, like a second electromagnetic wave which propagates through vacuum or air. This phase measurement can be performed by combining probe and reference beam to generate, depending on phase, constructive of destructive interference. Alternatively, a heterodyne phase detection scheme can be applied, in which the two waves which are brought to interference have slightly different frequency. Their interference results in a beat signal, whose envelope function is carrying the desired phase information. The beat frequency is determined by the frequency difference $\Delta \omega$ of the two waves, which can be set to values in the kilohertz to megahertz range to make the signal well accessible for measurement with the available detectors. The heterodyne phase detection concept can be applied to microwaves, throughout the terahertz range, and in the infrared and visible range and is sometimes referred to as "down-mixing" of the involved frequencies. For the generation of two waves with frequency difference $\Delta \omega$, different methods exist. One of them is to use two sources whose relative emission frequency is feedback-controlled, like two laser cavities ${ }^{1}$. Another method is to use only one source, split the beam in two and shift the frequency of one component. Frequency shifting is usually achieved via the Doppler effect and can be implemented e.g. with rotating optical gratings ${ }^{2}$ in the terahertz range, or with acousto-optic modulators (AOMs) in the visible and infrared range. On the twocolor interferometer used for plasma density measurement on the ASDEX Upgrade tokamak, whose optical layout we have discussed in ref. [3], AOMs are used to generate a frequency shift of $40 \mathrm{MHz}$. Accordingly, the readout electronics of the interferometer has to detect the relative phase of two $40 \mathrm{MHz}$ sinusoids, counting also integer multiples of $2 \pi$. There is a large variety of electronic circuits and data acquisition systems which fulfill this task, based on different operating principles. Just to name few of them, there are hard-wired phase counters which use an internal phase-locked loop and account for phase changes by increasing or decreasing the reading of a binary counter ${ }^{4}$. Another approach is to digitize the sinusoidal signal with an ADC whose sampling frequency is exactly $4 / 3$ of the signal frequency, resulting in intentional aliasing, and subsequent phase analysis by digital signal processing $^{5}$. In earlier work ${ }^{3}$, we have shown that digitization of probe and reference signal with a sufficient oversampling factor allows for carrying out the entire phase analysis by 
software, but requires fast ADCs and much memory if the beat frequency is high. The drawback of most of these methods is that they require either fast ADCs, with sampling frequencies of the order of the beat frequency, or even far above, which can be challenging when the beat frequency is in the upper $\mathrm{MHz}$ range, or they require sophisticated electronic circuits which have to be built specifically for the individual application. An example of the latter can be found in our earlier work ${ }^{6}$ and some of the references therein. Our recent approach, on the contrary, uses a simple circuit of low-cost components and a standard ADC.

\section{ELECTRONIC CIRCUIT}

The key component of our phase detector is the integrated gain and phase detector circuit AD83027, which has two inputs ("INPA" and "INPB") for sinusoidal signals, and a phase output ("VPHS") which (ideally) delivers $1.8 \mathrm{~V}$ when the input signals are in phase, $0 \mathrm{~V}$ when the input signals have a phase shift of 180 degrees, and varies by $10 \mathrm{mV}$ per degree in between. This output voltage will be referred to as U1 in the further discussion. The AD8302 does not show perfectly linear response over the full range from 0 to 180 degrees. According to figure 26 in the data sheet ${ }^{7}$, at $f=100 \mathrm{MHz}$ good linearity can be expected between about 30 and 150 degrees, while close to $0^{\circ}$ and $180^{\circ}$, the behavior is clearly nonlinear. We have experimentally characterized the actual response of the AD8302 circuit, as will be discussed below. Obviously, the output of a single AD8302 chip does in general not allow for unambiguous phase detection, as e.g. a voltage increase from 0 to $1.8 \mathrm{~V}$ could mean that phase has increased from 180 to 360 degrees, but could also mean that it has decreased from 180 to 0 degrees. Some phase detectors built from a single AD8302 chip have been reported in literature ${ }^{8}$. In the second reference, the dynamic range was restricted to the interval from $60^{\circ}$ to $120^{\circ}$, such that neither the phase ambiguity beyond $180^{\circ}$, nor the large nonlinearity close to $0^{\circ}$ and $180^{\circ}$ were a concern. For our multi-radian application, the phase ambiguity beyond 180 degrees has been resolved by splitting both sinusoidal input signals and feeding them into a second AD8302 chip, where the phase of one of the signals is shifted by 90 degrees, as illustrated in figure 1 and already briefly summarized in earlier work ${ }^{10}$ few years ago. The technique of signal splitting and shifting of one component by

90 degrees is equivalent to the very common method of quadrature phase detection ${ }^{11} 12$ 


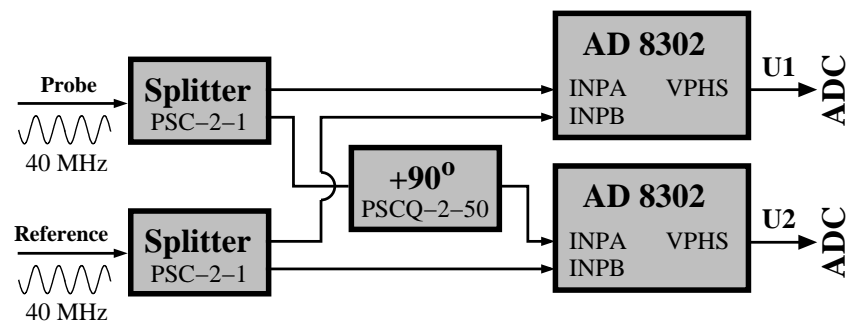

FIG. 1. Schematic block diagram of the phase counter circuit.

13. The classical approach of quadrature phase detection, however, uses mixers rather than AD8302 chips and therefore delivers output voltages that are proportional to the sine and the cosine of the relative phase of the input signals. Our circuit, on the contrary, delivers output voltages that depend (almost) linearly on the phase shift and do not depend on the amplitude of the input signals. The effort of implementation is clearly reduced by the usage of an AD8302 chip as compared to a mixer, as is discussed in the third section of ref. [9]. For signal splitting, MiniCircuits PSC-2-1 power splitters are used, and for phase shifting, the MiniCircuits PSCQ-2-50 is used. That way, an output voltage of the second chip is obtained which is (ideally) $0 \mathrm{~V}$ if the phase shift is 90 degrees and $1.8 \mathrm{~V}$ if it is 270 degrees. This voltage will be referred to as U2. The output of the two AD8302 chips, i.e. U1 and U2, is fed into a 2-channel simultaneous sampling ADC. In our application on the ASDEX Upgrade interferometer, the input frequency is $40 \mathrm{MHz}$ and the ADC sampling rate is 500 kSamples per second. We use a 14-bit ADC with an input voltage range of $-10 \mathrm{~V}$ to $+10 \mathrm{~V}$, which is more than actually needed for the $0-1.8 \mathrm{~V}$ signals, but was a convenient choice, as a multi-purpose ADC could be taken off-the-shelf. The choice of the ADC speed does not depend on the frequency of the sinusoidal input signals. Rather than this, it has to be matched to the time scale on which phase variations of the input signals occur.

\section{ANALYSIS ALGORITHM}

When analyzing the data recorded by the ADC, it was found that the AD8302 circuits exhibit imperfections. They do not accurately deliver $0 \mathrm{~V}$ when the input signals have 180 degrees phase shift, and not exactly $1.8 \mathrm{~V}$ when they are in phase. In the latter case, the output voltage was found to be close to $2 \mathrm{~V}$. This has been confirmed by an independent voltage measurement, to ensure it is not an artifact due to an offset or scale error of the ADC. 
It is not obvious from the AD8302 data sheet that the VPHS output voltage can exceed 1.8 V. The response of the AD8302 chips was confirmed not to be linear over the full range, i.e. when the phase shift of the input signals increases linearly in time, the output voltage does not linearly increase from its minimum to its maximum value. Whereas good linearity is observed in an interval of about $15^{\circ}$ to $165^{\circ}$, clear deviation from linearity occurs close to $0^{\circ}$ and $180^{\circ}$, which is in qualitative agreement with the corresponding figures 26-29 in the data sheet (that do not cover the case $f=40 \mathrm{MHz}$ ). To characterize the AD8302 response, we therefore connected two frequency generators to the inputs of the phase detector, whose internal clock oscillators were synchronized and whose output frequency was set to 40 and $40.0005 \mathrm{MHz}$, respectively, to generate a continuous phase drift. Ideally, the AD8302 should deliver a linear ramp up and down from 0 to $1.8 \mathrm{~V}$ and back under these conditions. The actual result for the two outputs (U1 and U2) of the phase counter unit is shown in figure 2. We built in total four phase counter units from eight AD8302 chips, which were found to show different response, as the second plot panel of figure 2 illustrates (grey dashed lines). From the data obtained with the two frequency generators, a calibration curve has been derived for each phase detector. The time axis was split equidistantly into 360 segments which correspond to a relative phase of $0,1,2, \ldots$ degrees. This was repeated for all oscillation periods in the calibration data set. Finally, the average voltages U1 and U2 for each integer angle (in degrees) were calculated, resulting in a calibration curve defined by 360 data points, which we label $\left(U 1_{i} \mid U 2_{i}\right), i \in\{0, \ldots, 359\}$, in the further discussion.

To perform phase measurements, the voltages U1 and U2 are recorded with the ADC. For each point in time, the phase $\varphi$ is then calculated with a computer algorithm, which works as follows: For the measured pair of voltages $(U 1 \mid U 2)$, the point $\mathrm{i}$ on the calibration curve is determined which is closest, i.e. which minimizes $d_{i}=\sqrt{\left(U 1-U 1_{i}\right)^{2}+\left(U 2-U 2_{i}\right)^{2}}$. After this, the distance $d_{j}$ to the second-closest point $\mathrm{j}$ is calculated, and linear interpolation is performed between the angles corresponding to those two points, using the ratio $d_{i} / d_{j}$ :

$$
\varphi\left[\text { in }^{o}\right]=i+(j-i) \frac{d_{i}}{d_{i}+d_{j}}
$$

This is illustrated in figure 3. In case of input signal perturbation, which we sometimes encountered as a result of electric cross-talk, the signals might no longer have proper sinusoidal shape, which often leads to data points $(U 1 \mid U 2)$ which are far off the calibration curve. In such cases, an outlier rejection algorithm was found to be helpful, which flags the result 

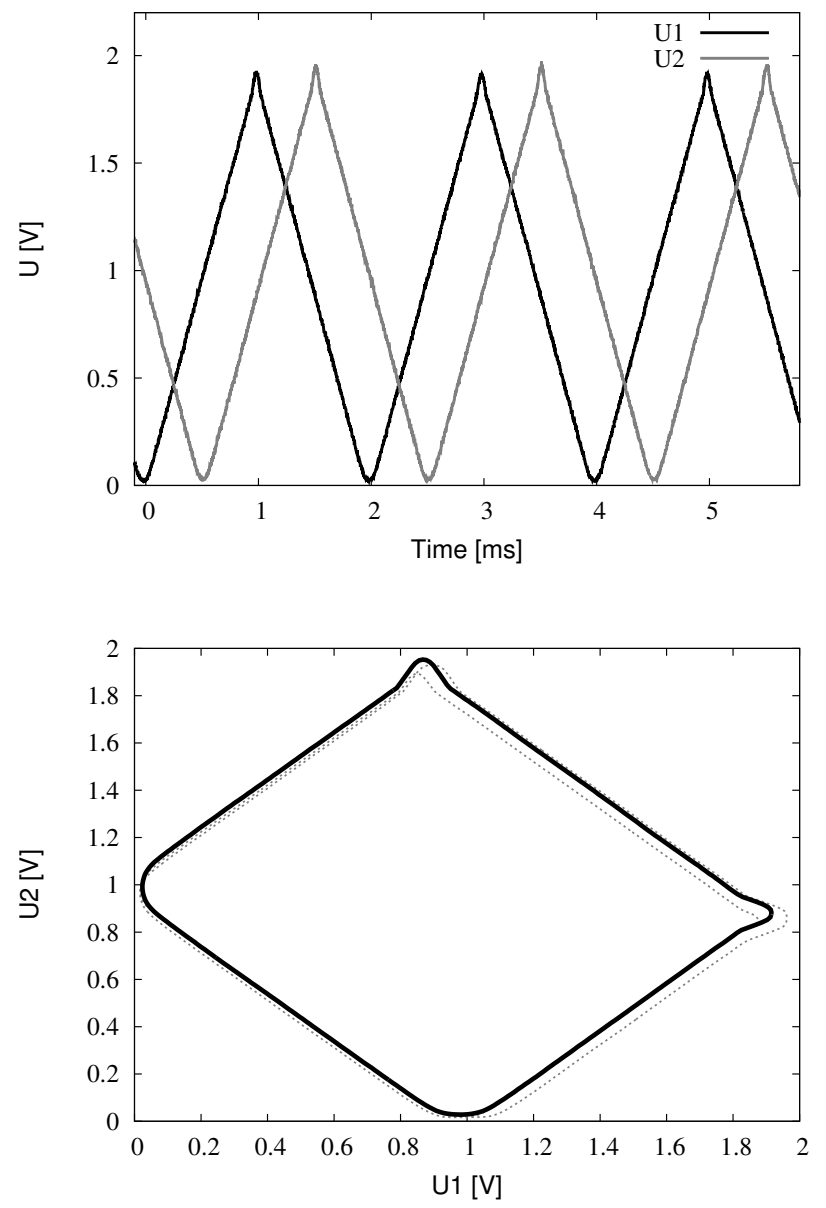

FIG. 2. Result of feeding two sinusoidal input signals of 40 and $40.0005 \mathrm{MHz}$ into the phase counting circuit. Top: Time traces of the output voltages U1 and U2. It can be seen that the upper reversal point is actually close to $2 \mathrm{~V}$, and slightly differs for U1 and U2. Bottom: Phase diagram, where U1 and U2 have been plotted versus each other. The bold black curve corresponds to the same phase counter shown in the upper plot panel. The dashed grey curves correspond to two other circuits that were built, illustrating the spread within a delivery batch of chips.

as invalid if $d_{i}$ exceeds an empirical threshold. This threshold should be of the order of the maximum distance between two neighboring points on the calibration curve, but must not be less than half of that value.

The approach described so far allows the determination of the phase shift as a fraction of $2 \pi$. Many applications require multi-radian phase detectors, which are capable of tracking phase shifts greater than $2 \pi$. This has been accomplished by introducing a variable for the integer multiples of $2 \pi$ in the analysis algorithm which is incremented by one when the 


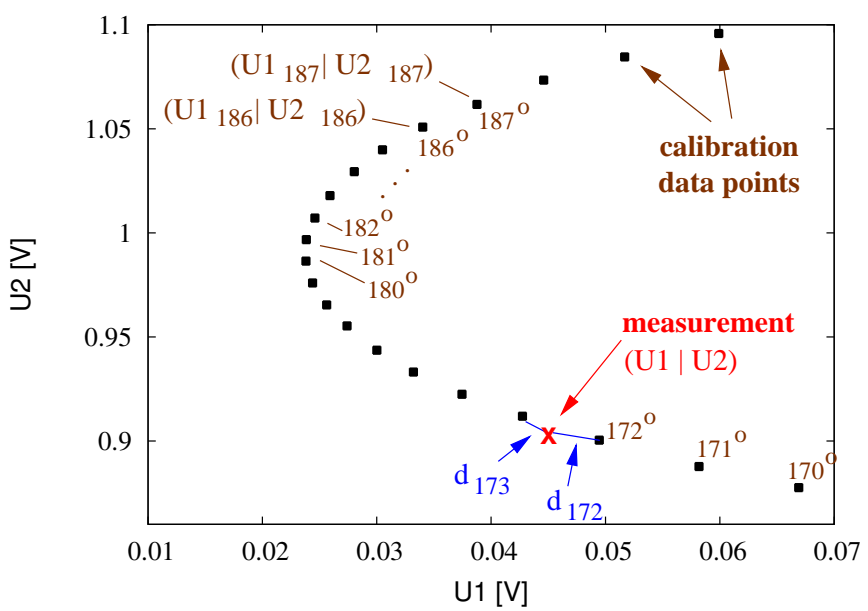

FIG. 3. Illustration of the interpolation algorithm: The phase diagram shows a part of the calibration curve, which consists of 360 data points - one per degree. To calculate the phase corresponding to a measured data point (U1 | U2), the closest and second-closest point on the calibration curve are determined - in the example, this is $\mathrm{i}=173$ and $\mathrm{j}=172$. The corresponding distances in the U1-U2 plane are labeled $d_{173}$ and $d_{172}$. In the example, the resulting angle is approximately $172.7^{\circ}$.

reconstructed phase jumps from 360 to 0 degrees, and which is decremented by one when there is a transition from 0 to 360 degrees. This requires the ADC sampling frequency to be chosen large enough to ensure that phase changes in between two samples will be much less than 180 degrees under all experimental conditions, as stated at the end of section II.

\section{RESULTS}

The two-color interferometer on the ASDEX Upgrade tokamak sends two laser beams from a helium-neon $\left(\lambda_{\mathrm{HeNe}}=633 \mathrm{~nm}\right)$ and a carbon dioxide $\left(\lambda_{\mathrm{CO} 2}=10.6 \mu \mathrm{m}\right)$ laser through a plasma along the same path ${ }^{3}$. The total optical path that the laser beams travel from the AOMs to the detectors is more than 10 meters. Although the interferometer frame rests on an independent support structure on the concrete floor of the experiment hall, the presence of vacuum pumps and other devices with moving parts inside causes a certain level of vibration in the optical setup. The two-color setup measures two phase shifts experienced by two different laser wavelengths, which allows one to compensate for vibrations and to get an unbiased measurement of the line-integrated electron density in the plasma. First, for simplicity, we discuss only results from the HeNe branch of the two-color interferometer. 

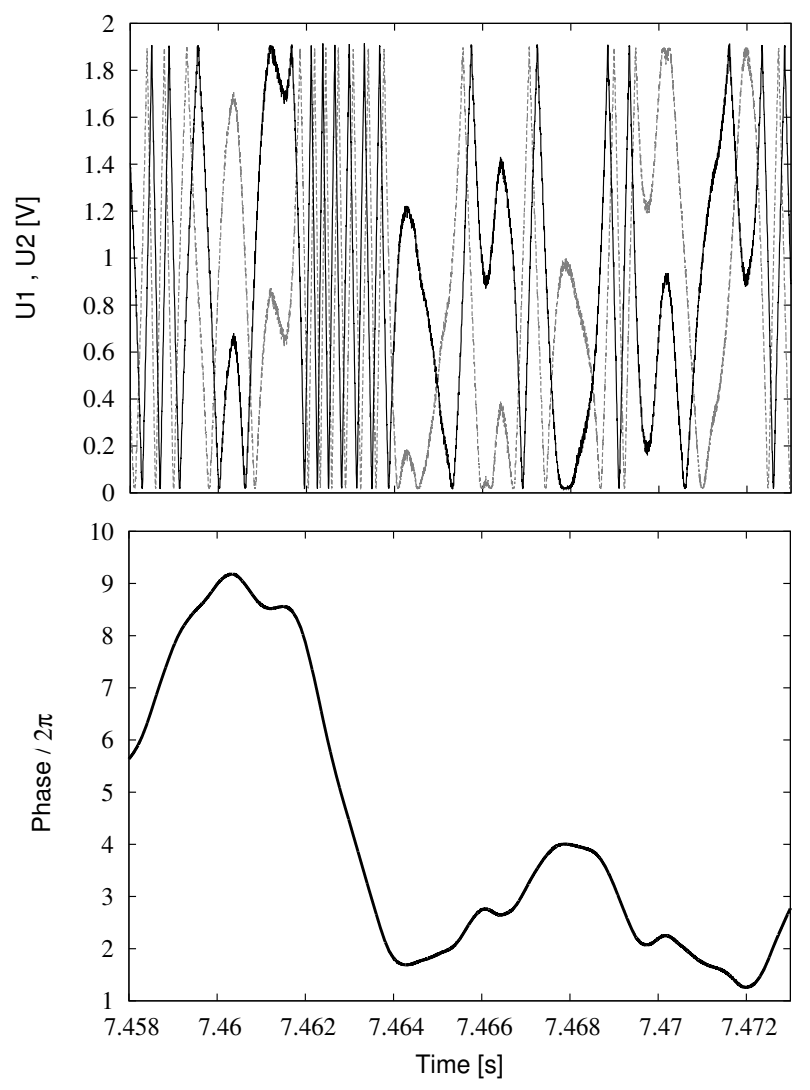

FIG. 4. Temporal evolution of the voltages U1 (black) and U2 (gray, upper plot panel) and the reconstructed phase evolution (lower plot panel) for the helium-neon branch of the central interferometer channel in ASDEX Upgrade discharge \#32214. The diagram shows a short time window of about $15 \mathrm{~ms}$ out of an 8-second plasma discharge.

Phase changes experienced by the HeNe beam are dominantly caused by vibrations, whereas the plasma density has only little influence. As discussed above, the interferometer uses heterodyne detection, where an acousto-optic modulator shifts the frequency of one beam component by $40 \mathrm{MHz}$. The resulting interference signal is a $40 \mathrm{MHz}$ beat signal, which is registered by a fast photo diode. The phase counting electronics compares the phase of this detector signal to the phase of the modulator driver signal, which is a $40 \mathrm{MHz}$ sinusoid as well. Figure 4 shows the temporal evolution of the phase counter output voltages $\mathrm{U} 1$ and U2, as well as the phase evolution reconstructed by the algorithm described in the previous section. The maximum phase variation in this example is about $8 \times 2 \pi$ peak-to-peak, corresponding to an optical path change of about 5 micrometers. Figure 5 finally shows an example of a plasma density measurement carried out with the new phase counting electronics. Two 
of the phase counters have been installed on the central interferometer chord to record both, the phase evolution of the HeNe and the $\mathrm{CO}_{2}$ branch of the interferometer. The figure shows the corresponding time traces over a time period of 3 seconds. It can be seen that the two traces look very similar, representing mainly the optical path changes in the interferometer due to vibrations. In accordance with the ratio of the wavelengths, the HeNe branch registers phase changes of more than $70 \mathrm{x} 2 \pi$, whereas the phase in the $\mathrm{CO}_{2}$ branch varies by only about $4 \mathrm{x} 2 \pi$ (not considering the negative-going spike at $\mathrm{t}=2.3 \mathrm{~s}$, which is not caused by vibration, but by a high plasma density event, as will become apparent in the further discussion). Electron density information is recovered from the two phases measured via equation (2) in ref. [3], which implies that basically the HeNe phase is multiplied by the wavelength ratio and subtracted from the $\mathrm{CO}_{2}$ phase. With the appropriate pre-factor and sign, this yields the time trace of the line-integrated electron density, which is also shown in figure 5. The corresponding plasma discharge was shut down by massive gas injection at $\mathrm{t}=2.3 \mathrm{~s}$, which is a common method of rapidly terminating a tokamak plasma. This technique was developed for unstable discharges, where the forced shutdown of the discharge reduces both, thermal loads to the plasma-facing components and electromagnetic forces on the plasma vessel, as compared to the uncontrolled collapse of the discharge, which otherwise would occur shortly after. The massive gas injection induces a sudden and steep rise of the electron density up to a value of about $6.310^{20} \mathrm{~m}^{-2}$ within roughly a millisecond, followed by a decay down to zero within the next 15-20 ms, after which there is no plasma any more. Even under these conditions, the multiples of $2 \pi$ have been correctly tracked by the phase counter, such that the time trace returns to zero at the end. The sudden disruption of the toroidal plasma current caused by the gas injection results in substantial currents induced in the vacuum vessel structure, which cause electromagnetic forces acting on the tokamak. Although mechanically coupled only via the massive concrete floor, vibrations are also excited in the interferometer structure, which explains the strong oscillation of both phase signals after $t=2.3 \mathrm{~s}$. The density trace remains unaffected, as the two-color interferometer successfully compensates the vibrations.

Over several years of operation, the phase counters have been found to be reliable and stable in their calibration curves. Failure of the tracking of multiples of $2 \pi$ is only observed when the input signals have too little amplitude or no longer proper sinusoidal shape. The former may happen in case of optics misalignment, or temporary beam refraction on the 


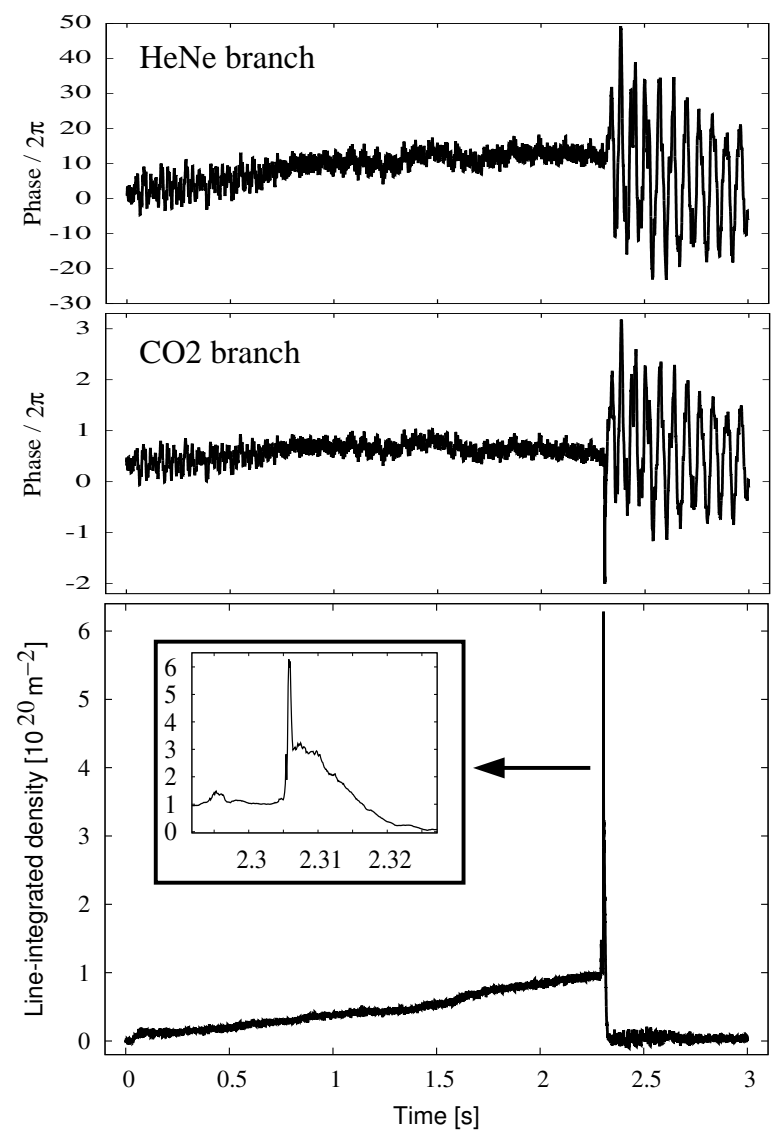

FIG. 5. Temporal evolution of the phase in the HeNe branch (top) and the $\mathrm{CO}_{2}$ branch (center) during ASDEX Upgrade discharge \#33242, which was shut down by massive gas injection at $\mathrm{t}=2.3 \mathrm{~s}$. The line-integrated electron density calculated from those two phase signals is shown in the lower plot panel.

$\mathrm{CO}_{2}$ branch when large quantities of gas are injected to shut down the discharge, like in the example presented in ref. [3]. The latter is regularly observed when ion cyclotron resonance heating at a frequency of $36.5 \mathrm{MHz}$ is applied on ASDEX Upgrade, which electrically crosstalks into the $40 \mathrm{MHz}$ interferometer signals despite of shielding efforts. The installation of band-pass filters to reject the $36.5 \mathrm{MHz}$ helped to clearly mitigate this problem.

\section{SUMMARY}

In this article, we have presented a rather simple phase detection circuit, which requires only few commercially available electronic components and an ADC with moderate speed and resolution, allowing for fast implementation at low cost. It is applicable to a wide range 
of frequencies, with an upper limit of $2.7 \mathrm{GHz}$ set by the capabilities of the AD8302 chip. The signal splitter and $90^{\circ}$ phase shifter have to be appropriately chosen for the frequency range of interest, with the choice given in section II yielding good results at the $40 \mathrm{MHz}$ beat frequency of the ASDEX Upgrade interferometer. Since the integrated circuits used for phase detection show non-linear response, with the exact characteristic varying from chip to chip, an initial calibration measurement needs to be carried out to obtain a calibration curve for each single phase detector unit. We have used the calibration data set over years and have not observed any noticeable drift in time. An algorithm has been presented which calculates the phase information from the digitized output signals of the phase detector units. In our application, data analysis is performed offline. At the sampling frequency of $500 \mathrm{kSamples}$ per second, however, the algorithm is less than a factor of 3 too slow for real-time application on a standard CPU, where it takes roughly 25 seconds to process the data of a 10-second plasma discharge. By further optimization of the algorithm for speed, e.g. by using lookup tables rather than performing calculations, or implementation on a programmable logic device, real-time capability appears achievable.

Due to the simplicity of the involved hardware, this phase counter could be an interesting option for many applications in and beyond interferometry, and for scientific research applications was well as for academic education purposes. Implementing the hardware part is simple, cheap and straight-forward. With the initial knowledge that phase counter response is non-linear and the recording of a calibration data set is required, also the implementation on the software side can be accomplished with moderate effort.

\section{ACKNOWLEDGMENTS}

This research project received funding from the former European Fusion Development Agreement (EFDA) from February 2012 till March 2013 under grant No. WP12-IPH-A071-2-05/PS-01/IPP. In addition, this work has been carried out within the framework of the EUROfusion Consortium and has received funding from the Euratom research and training programme 2014-2018 under grant agreement No 633053. The views and opinions expressed herein do not necessarily reflect those of the European Commission. 


\section{REFERENCES}

${ }^{1}$ K. Kawahata, A. Ejiri, K. Tanaka et al., Fusion Engineering and Design 34-35, 393-397 (1997).

${ }^{2}$ D. Veron, Optics Communications 10, 95-98 (1974).

${ }^{3}$ A. Mlynek, G. Pautasso, M. Maraschek et al., Fusion Science and Technology 61, 290-300 (2012).

${ }^{4}$ H. R. Koslowski, Measurement Science and Technology 5, 307 (1994).

${ }^{5}$ M. A. Van Zeeland, R. L. Boivin, T. N. Carlstrom et al., Review of Scientific Instruments 79, 10E719 (2008).

${ }^{6}$ A. Mlynek, G. Schramm, H. Eixenberger et al., Review of Scientific Instruments 81, 033507 (2010).

${ }^{7}$ Data Sheet of AD8302, Analog Devices, Rev. A, (2002).

${ }^{8}$ N. Strauss, I. Ernsting, S. Schiller et al., Applied Physics B 88, 21-28 (2007).

${ }^{9}$ Y. F. Yee, C. K. Chakrabarty, Measurement 40, 849-853 (2007).

${ }^{10}$ A. Mlynek, L. Casali, H. Eixenberger et al., 40th EPS conference on plasma physics, Espoo, Finland, Europhysics Conference Abstracts 37D, P6.012 (2013) http://ocs.ciemat.es/EPS2013PAP/pdf/P6.012.pdf.

${ }^{11}$ S. Vergamota, L. Cupido, M. E. Manso et al., Review of Scientific Instruments 66, 25472551 (1995).

${ }^{12}$ A. R. Jacobson, Review of Scientific Instruments 53, 918-919 (1982).

${ }^{13}$ N. B. Yim, C. I. Eom, S. W. Kim, Measurement Science and Technology 11, 1131-1137 (2000). 